\title{
Oncogenomics: a personalized cancer therapy
}

\begin{abstract}
This article is concerned with the generally small proportion of genetically-influenced cancers in which the environment plays no role. After defining the nature of cancer and its clonal evolution, I review the various cancer theories and corresponding therapies that have evolved over the last several decades. In this context, I also discuss the problem of drug resistance that frequently develops in patients undergoing these cancer treatments. I then systematically review the several major recent developments in cancer treatment. I finally introduce the newest oncogenomic approach that seems to offer considerable promise, at least for solid tumors. This latter approach requires scanning the genome of individual patients to sift out "actionable findings" from DNA sequencing data, a long and tedious task in need of acceleration if we hope to implement at last personalized cancer therapies. I suggest looking also beyond copy number variations in the search for those actionable findings.
\end{abstract}

Volume 9 Issue $6-2018$

\author{
Alain L Fymat \\ International Institute of Medicine and Science, California, USA
}

Correspondence: Alain L Fymat, International Institute of Medicine and Science, California, USA, Tel (760) 507-6862, Email alain.fynat@fiimas.org

Received: November 22, 2018 | Published: November 28, 2018

Keywords: oncogenomics, clonal cancer, cancer theories, drug resistance, cancer treatments

Abbreviations: BBB, Blood Brain Barrier; CAR, Chimeric Antigen Receptor; GBM, Glio Blastoma Multiform; HCGP, Human Cancer Genome Project; HGP, Human Genome Project; NCT, Nano Chemo Therapy; ND, Nano Devices; NP, Nano Particles; PD, Programmed Death; TPG, Tumor Promoter Genes; TSG, Tumor Suppressor Genes

Diseases listed: Anemia (perniciousness, Indian); Cancer (breast, cervix, colon, lung, prostate, testes); Carcinoma (adeno, basal cell, spindle cell, squamous cell); Glioma; Glioblastoma; Hodgkin disease; Leukemia (acute lymphoblastic, acute myeloid); Lymphoma (Burkitt); Melanoma; Retinoblastoma; Sarcoma (bone, Kaposi, spindle cell)

Drugs cited: Adriamycin, Aminopterin, Bleomycin, Carakinumab, Carfizomib, Cisplatin, Daunorubicin, Dexomethasone, Etoposide, Ibrance, Paclitaxel, Pembrolizumab (Keytruda), Phenantidine, Trametinib, Venetoclax, Vinblastin

\section{Introduction}

The vast majority of cancer cases are due to environmental risk factors, many of which (but not all) being controllable lifestyle choices and thus preventable. It has been suggested that more than $30 \%$ of cancer deaths could be prevented by avoiding risk factors including: tobacco, overweight, obesity, insufficient or/and inappropriate diet, physical inactivity, alcohol, transmitted infections, and air and water pollution. However, not all environmental causes are controllable such as naturally occurring background electromagnetic radiation. This article is concerned with those cancers that are due to genetics. In a companion article, I will discuss the case of cancers caused by epigenetics and ecogenetics and their oncological treatments (which I shall refer to as oncoepigenomics and oncoecogenomics, respectively).

\section{On the nature of cancer and its clonal evolution}

Many diseases are lumped together under the denomination "cancer" because they share a fundamental biological feature, namely abnormal cell growth. However, cancer is not a single disease but a multiplicity of diseases caused by the uncontrolled growth of a single cell. It is fundamentally a disease of tissue growth regulation failure unleashed by mutations, which are changes in DNA that specifically affect genes and incite unlimited growth. We naively thought that cancer could be defeated by either preventing mutations from occurring in normal cells or else finding the means to eliminate the mutated cells without compromising normal growth. Unfortunately, this view does not take into account the perverse genetic intertwining of normal and cancerous growths. Cancer is woven into our genome as the mutated genes are but distorted versions of the normal ones; they are braided together and unbraiding them is the most formidable undertaking.

In order for a normal cell to transform into a cancer cell, the genes that regulate cell growth and differentiation must be altered. The affected genes are divided into two broad categories: tumor promoter genes (TPG) or oncogenes that promote cell growth and reproduction, and tumor suppressor genes (TSG). That inhibit cell division and survival. Malignant transformation can occur through the formation of new oncogenes, the inappropriate over-expression of TPG, or else by the under-expression or disabling of TSG. Cancer cells can grow faster, flourish more profusely, adapt better, recover more rapidly, and repair faster... than normal cells. They are in effect more perfect versions of normal cells......and can even become immortal. Typically, changes in many genes are required to transform a normal cell into a cancer cell.

Genetic changes can occur at different levels and by different mechanisms. The gain or loss of an entire chromosome can occur through errors in mitosis. More common are mutations, which are changes in the nucleotide sequence of genomic DNA. Large-scale mutations involve the deletion or gain of a portion of a chromosome. Genomic amplification occurs when a cell gains many copies (often 20 or more) of a small chromosomal locus, usually containing one or more oncogenes and adjacent genetic material. Genomic translocation occurs when two separate chromosomal regions become abnormally fused, often at a characteristic location. Small-scale mutations include point mutations, deletions, and insertions, which may occur in the promoter region of a gene and affect its expression, or may occur 
in the gene's coding and alter the function or stability of its protein product. Disruption of a single gene may also result from integration of genomic material from a DNA virus or retrovirus, leading to the expression of viral oncogenes in the affected cell and its descendants. Replication of the enormous amount of data contained within the DNA of living cells will probabilistically result in some errors (mutations). Complex error correction and prevention are built into the process to safeguard the cell against cancer. However, if a significant error occurs, the damaged cell can "self-destruct" through programmed cell death (apoptosis). If the error control processes fail, then the mutations will survive and be passed along to daughter cells.

Some environments make errors more likely to arise and propagate. Such environments can include the presence of disruptive substances called carcinogens, repeated physical injury, heat, ionizing radiation, or hypoxia. The errors that cause cancer, are selfamplifying and compounding. For example: a mutation in the errorcorrecting machinery of a cell might cause that cell and its daughters to accumulate errors more rapidly; a further mutation in an oncogene might cause the cell to reproduce more rapidly and more frequently than its normal counterparts; another mutation may cause loss of a TSG, disrupting the apoptosis signaling pathway and resulting in the cell becoming immortal; yet, another mutation in the signaling machinery of the cell might send error-causing signals to nearby cells.

The transformation of normal cells into cancer is akin to a chain reaction caused by initial errors, which compound into more severe errors, each progressively allowing the cell to escape the controls that limit normal tissue growth. This rebellion-like scenario becomes an undesirable survival of the fittest, where the driving forces of evolution work against the body's design and enforcement of order. Once cancer has begun to develop, this ongoing process (termed "clonal evolution") drives progression towards more invasive stages. Clonal evolution leads to intra-tumor heterogeneity that complicates designing effective treatment strategies.

\section{Cancer theories and corresponding therapies}

Various hypotheses (theories) of cancer and corresponding therapies were propounded over time:

a) Blood suppuration: John Bennett (erroneously) characterized leukemia as a blood suppuration (1845). This was disproved by Michael Anton Bermer who described that illness as neoplasia.. not a suppuration of blood (1860). William H. Coley, James Ewing and Ernest Codman treated bone sarcoma with a mixture of bacterial toxins (the so-called "Coley toxin") but with unpredictable results due probably to immune stimulation, highlighting the important role of the immune system in cancer therapy.

b) Somatic mutation: The somatic mutation hypothesis posited that environmental carcinogens (soot, smoke, radium, X-rays, etc.) permanently altered the cell and thus caused cancer (early $20^{\text {th }}$ century)

c) Viral propagation and infection: In 1910, experimenting with spindle-cell sarcoma, Peyton Rous concluded that the cause of cancer was not a cell or an environmental carcinogen but rather some tiny particle within a cell...a virus, later called "Rous sarcoma virus" (RSV). As an endogenous causative factor, RSV dealt a deep blow to the exogenous somatic mutation theory. In 1948, Rous reinforced the conclusion of a virus as the cause of cancer and Denis Burkitt concluded that "Burkitt lymphoma" (an aggressive form of lymphoma) was caused by a human virus: the Epstein-Barr virus (EBV) that is responsible for infectious mononucleosis. The notion that cancer was an infectious disease was then resurrected (as hypothesized in the blood suppuration hypothesis), but this condemned the somatic mutation theory of cancer to its death. By the mid-1970s, a revival of the viral origin theory took place.

d) Retroviral propagation: Howard Temin had disproved the central "dogma" (a misnomer at best; rather a hypothesis) of molecular biology which posited a unidirectional flow of information: DNA $\rightarrow$ RNA (so-called transcription) $\rightarrow$ proteins. Rather, viruses possessed the reverse capacity: RNA $\rightarrow$ DNA $\rightarrow$ (so-called reverse transcription). Thus, a cancercausing virus could become a physical part of a cell's genes. This and like viruses (or retroviruses) have genes that exist as RNA outside cells. When these RNA viruses infect cells, they make a DNA copy of their genes and attach this copy to the cell's genes. This DNA copy, called a provirus makes RNA copies, and the virus is regenerated to form new viruses ad infinitum, unleashing pathological mitosis, that is cancer. Unfortunately, retroviruses are not the cause of cancer but of another disease - HIV.

e) Anti-vitamin and the beginning of chemotherapy: Before the advent of modern imaging techniques, it was not possible to quantify cancer and measure its progression, the only exception being for leukemia. In leukemia, a simple blood draw and its analysis could inform on the white blood cell count and its changes as a result of drug treatment. Sidney Farber was kindled by two contemporaneous hematological findings; (a) George Minot's discovery that "pernicious" anemia was caused by a lack of vitamin $B_{12}$ and could be treated by administrating that vitamin, and (b) Lucy Wills' treatment of "Indian-workers" anemia by the vitamin-like folic acid or folate (a crucial DNA building block). He was likewise kindled by the link between vitamins, bone marrow and normal blood. Unfortunately, neither of these two vitamins could treat cancer (actually, quite the contrary in the case of folic acid) so Farber searched for "anti-vitamins" (which do not exist). Nonetheless, this was the beginning of the search for a chemical to treat cancer, that is "chemotherapy". In the 1920s, while working for an antidote to the anemia of folate deficiency, Yellapragada Subbarao developed decoy molecular structures that nearly mimicked natural molecules. These structures can bind to enzymes and receptors and block their actions. They behave like antagonists to folic acid (anti-folates),...that is the very anti-vitamins Farber was looking for. In 1947-8, Farber successfully treated, at least temporarily, a patient presenting with acute lymphoblastic leukemia (ALL) with the antifolate drug aminopterin supplied to him by Subbarao - an unprecedented remission in the history of leukemia. Unfortunately, the remission was short-lived (a few months) and the leukemia came roaring back. This could also truly be thought of as the beginning of chemotherapy the disappearance (even though temporary) of an aggressive systemic cancer via a chemical drug.

f) Combination chemotherapy: Particular combinations of cytotoxic drugs would cure the cancer, which marked a new beginning of chemotherapy. In 1974, the survival rate from metastatic testes cancer was less than $5 \%$. With a threedrug cocktail called BVP (bleomycin, vinblastine, cisplatin abbreviated P for platinum), Larry Einhorn cured this solid cancer. The notion that even relatively indiscriminate cytotoxic 
agents discovered largely by accident would cure cancer captivated oncology. An avalanche of such drugs poured in: taxol, adriamycin, etoposide, bleomycin (an antibiotic), and an alphabet soup of other combinations (ABVD, BEP, C-POMP, ChlaVIP, CHOP, ACT). At about the same time, using a high dose combination chemotherapy (a cocktail of seven drugs), Ian Magrath and John Ziegler cured Burkitt lymphoma. Yet, despite the escalation of drugs and doses, the efficacy of the drug regimen remained minimal. The pattern repeated itself regularly for many forms of cancer. Over the decades that followed, a large array of drugs were synthesized for the fight on cancer. Hodgkin disease is now cured with multi-drug chemotherapy; locally advanced lung cancer is controlled with the triadic treatment (surgery + chemotherapy + radiation); lymphoblastic leukemia can be induced into a prolonged remission after intensive chemotherapy with cure rates of $80 \%$ routinely achieved. The mortality for every form of cancer (lung, breast, colon,. prostate, etc.) has continuously dropped for fifteen straight years. For some other cancers (colon, cervix), the decline is almost certainly due to the success of secondary prevention - cancer screening. For leukemia, lymphoma, and testicular cancer, the decline is the result of successful chemotherapeutic treatments. The death rate for breast cancer has been dramatically brought down by the combination (mammography + surgery + adjuvant chemotherapy).

g) Proto-oncogene: In the mid-1970s, Harold Varmus and J. Michael Bishop showed that a precursor of a cancer-causing gene - which they called the "proto-oncogene", was a normal cellular gene existing inside cancer cells. Mutations induced by chemicals or X-rays caused cancer, not by inserting foreign genes, but by activating such endogenous proto-oncogenes. Thus, cancer genes come from within the human genome.

h) Two-hit hypothesis: In the early 1970s, Alfred Knudson proposed the two-hit hypothesis of cancer, initially for retinoblastoma. He proposed that genes come in two flavors: "positive" genes that are mutant-activated versions of normal cellular genes (they accelerate cell division, but only when the cell receives an appropriate growth signal) and "negative" genes that suppress cell division when the cell receives appropriate signals, having been inactivated by mutations. Both abnormalities activate proto-oncogenes and inactivate tumor-suppressors. When this activation/inactivation falls out of equilibrium, cancer then develops.

i) Metastatic mechanism: In developing cancer, individuals differ in both their inherited tendency and exposure to the environment, a multi-event combination process. At the cellular level, mutations occasionally occur as the cells divide and, although not heritable by any offspring (somatic mutations), they can affect cell behavior sometimes causing more frequent growth and division. Normally, cell division responds to growth factors and stops when encountering growth inhibitory signals from surrounding cells. After a number of divisions, the cell dies and remains within the epithelium from which it is unable to migrate to other organs. To become cancerous, it would have to bypass these signals and accumulate new genetic mutations in a number of genes (3-7), the most frequent being a loss of function of the $\mathrm{p} 53$ protein (a tumor suppressor) or in the $\mathrm{p} 5$ pathway and/or a gain of function through mutations in the protein or in other oncogenes. It will then keep growing, escape from the epithelium and the primary tumor, cross the endothelium of a blood vessel, be transported by the blood stream and colonize (a) new organ(s) to give rise to metastases.

Thus, after a circuitous detour through several hypotheses and theories (elements of which are nonetheless valuable), we have now come to the conclusion that cancer is stitched into our genome; oncogenes arise from mutations in essential genes that regulate the growth of cells and accumulate in these genes when DNA is damaged by carcinogens (theoretically preventable), but also by seemingly random errors in copying genes when cells divide (a flaw deeply entrenched in ourselves and therefore unpreventable). Thus, we can rid ourselves of cancer only in as much as we can rid ourselves of the processes in our physiology that depend on growth - aging, regeneration, healing, and reproduction.

\section{How cancr cells develop drug resistance}

While many types of chemotherapy have been developed against cancer, oncologists do not know before starting treatment whether a patient might benefit from a particular drug. So being able to identify through a laboratory test whether a patient's tumor is either resistant or sensitive to a specific drug is crucial to enabling the rapidly developing field of "personalized medicine".

In a breakthrough discovery, Prof. Karni and his team of researchers at the Hebrew University Medical Center, Jerusalem, Israel, have identified a process by which cancer cells become resistant to certain drugs. This process will lead to a reliable prediction as to which patients will be helped by chemotherapy and recover, and which patients will not be helped by these drugs. This finding could enable the reversal of the process and inhibit metastasis of malignant tumor cells. The researchers found that breast, lung and colon cancer cells change the structure of an enzyme called mnk-2, which is involved in the transmission of information from the environment/ body into the cell. They also showed that the enzyme has two forms, a "normal" one that inhibits cancer and another form that promotes cancer development. They further showed that cancer cells change the structure of the mnk-2, so they eliminate the form that inhibits cancer and elevate the form that induces it, thus allowing the cancer cells to survive and grow faster. Still further, they found that the anticancer form of the enzyme activates a program of apoptosis (suicide) in normal cells under stress conditions.

To fight the resistance process, the Karni team developed molecules that can convert the cancerous form of the mnk-2 enzyme back into its normal form so they become sensitive to stress and to absorbing chemotherapeutic drugs. More importantly, the molecules that change the cancerous form of mnk-2 into the normal form will make it possible to overcome the drug resistance of cancer cells, making them instead sensitive and responsive to various anti-cancer treatments. Further laboratory work on this aspect is continuing.

The above mechanism explains how cancer cells eliminate the anti-cancer form of mnk-2 without changing their DNA and how they become resistant to anti-cancer treatments, a problem that exists for almost every cancer treatment today. The new molecules they developed to change the structure of the mnk- 2 enzyme back to its normal form will enable re-sensitizing cancer cells to anti-cancer therapies. This research could lead to the development of a new biomarker for testing the sensitivity of a patient to specific drugs. The possibility of examining whether a patient will benefit from a specific drug treatment before the treatment starts is of primary medical interest. The Israeli scientists are now developing a diagnostic test for the marker they found. 


\section{Major recent developments in cancer treatment} below:

The major recent developments in cancer treatment are recounted

a) Innate immunotherapy with neutrophil-mediated drug delivery: A neutrophil-mediated anticancer nanotechnological drug delivery has been proposed for the suppression of postoperative malignant glioma recurrence. Neutrophils are white blood cells in the granulocytic series of blood cell development Formed by myelopoietic tissue of the bone marrow and released into the circulating blood, they can penetrate inflamed brain tumours and, although they are not typically attracted to glioblastomas (GBMs), they are recruited at sites where tumors had been removed in response to post-operative inflammation. Liposome capsules have been developed that encased Paclitaxel (PTX), a traditional chemotherapy drug, with lipids, loaded into neutrophils and injected in the blood. The neutrophil-carrying drugs are able to penetrate the blood nbrain barrier (BBB), destroy residual cancer cells, and slow the growth of new tumors. Inflammatory factors released after tumour resection guide the movement of the neutrophils into the inflamed brain. The highly concentrated inflammatory signals in the brain trigger the release of liposomal PTX from the neutrophils, allowing delivery of PTX into the remaining invading tumour cells. This neutrophil-mediated delivery of drugs efficiently slows the recurrent growth of tumours, significantly improving survival rates, but does not completely inhibit the regrowth of tumours. While tumor recurrence is not completely prevented, overall, this treatment prolong life significantly. The technique can be used in other inflammation-mediated disorders, and any other diseases that naturally attract neutrophils.

b) Synthetic immunotherapy: Immunotherapy represents a paradigm shift in cancer treatment in that it targets the immune system, not the cancer itself. In 2013, the Science magazine declared it as that year's breakthrough! Nonetheless, today's immunotherapies do not help every one (e.g., the odds remain long for patients with metastatic cancer) and biomarkers that might offer answers remain to be designed as well as experimenting with ways to make therapies more potent. In clinical trials, new immune system-boosting cancer drugs have saved lives in seemingly untreatable melanoma or lung cancer cases, but the drugs seem useless against colon cancer. Nonetheless, even cancers impervious to the new drugs (3\%$4 \%$ ) could be treated if those malignancies have the right error-riddled DNA signature. The idea of boosting the body's own defenses is elegant and appealing. Further, once their task completed, the engineered cells remain in the body, offering protection against recurrence or re-infection for years to come. Immunotherapy can use either natural or synthetic chimeric antigen receptors (CAR). We distinguish the following:

c) PD-1 inhibition: Tumor cells hide from T-cells by activating PD-1 receptors on the surface of the immune system's T- cells and can attack them if the receptors are blocked by PD-1 inhibitors. There are two new types of drugs (including pembrolizumab [Keytruda]) to harness the immune system, keeping tumors at bay for years. In clinical trials, they generally have worked in less than half the cases, and work best on tumors with lots of mutations. Advanced cancers seem more likely to respond if they have so-called mismatch repair mutations, which explains why the best outcomes have been for the heavy mutation tumors of lung cancer and melanoma. The hypothesis is that some of these mutations may alter genes to code for abnormal (or foreign) proteins or antigens so that the more of them, the more antigens to launch an attack from T-cells unleashed by a PD-1 inhibitor. Clinical trials found that mutations in mismatch repair genes can lead to cancerpromoting mutations and would respond to PD-1 inhibitors. In the case of few mutations, one implication is that the tumors might respond better to PD-1 inhibitors if they first receive radiation or chemotherapy that create new mutations.

d) Chimeric Antigen Receptor T-cells: Combining gene therapy, synthetic biology and cell biology, engineering T-cells involves the following steps: (a) Extracting from the blood T-cells known to respond best to a given disease; (b) implanting them with new genes using a custom-built virus; (c) creating cells that target a molecule (CD19) found on surfaces of few cancers; and (d) returning to the body the modified cells where their new DNA gives them a fresh set of targets to attack. The technique can be refined by overcoming the treatment's toxic effects that may occur in most advanced cancers. Here, a runaway reaction (called a cytokine storm) can be fatal so the dose is brought down to its lowest to reduce this immune system's overreaction. Unfortunately, besides CD19, we know of no other chemical target that is specific to cancer alone. A modified technique has been proposed in which cells are tweaked to attack when they sense not one but two different target chemicals. The idea is that whereas neither target may be unique, the combination might be, allowing the immune system to be unleashed on tumors whilst sparing healthy tissue. Engineered T-cells (and perhaps also B-cells, another part of the immune system) might be used to treat a wide range of diseases besides cancer, including HIV, immune deficiencies, and autoimmune disorders.

e) DNA Origami/Trojan: In the original origami technique, to foil drug resistance in solid tumors, a cancer drug is packaged in a capsule made of folded-up DNA. A chemotherapeutic drug [daunorubicin in the case of acute myeloid leukemia (AML)] upon entering cancerous cells recognizes them and pumps them back out through openings in the cell wall. In its refined version, the genome of a common bacteriophage and synthetic strands that were designed to fold up its DNA are encapsulated and do not encode any proteins or do any of the normal DNA functions. Potentially, the technique should work on most any form of drug-resistant cancer.

f) mnk-2 conversion to overcome drug resistance: Breast, lung and colon cancer cells change the structure of the enzyme $m n k-2$, which is involved in the transmission of information from the environment/body into the cell. This enzyme is binary with a "normal" form that inhibits cancer development and an "abnormal" form that promotes it. The balance between these two forms will determine whether the cancer is arrested or promoted. To overcome drug resistance, molecules have been developed that can convert the abnormal to the normal form of mnk-2. The underlying mechanism elucidates how cancer cells eliminate the anti-cancer form, and provides a means to reverse it.

g) Antiangiogenesis: Cancer cells hijack and feed off blood vessels. In the brain, this results in a weakened BBB and this may provide one reason for the rapid spread of GBMs. This observation may lead to new ways to kill brain tumors using the BBB weakness to get targeted drugs into the brain during the early stages of the cancer. Outside the main tumor mass, nearly all GBM cells gather in the space between the astrocytic 
end feet and the outer surface of blood vessels. The cancer cells use the network of small blood vessels as a scaffold to guide their migration along the blood vessels as they extract nutrients from the blood inside. The GBM cells hijack control over blood flow in the BBB away from the astrocytes, loosening the tight junctions, and resulting in a breakdown in the barrier. Very small groups of GBM cells - even individual cells could weaken the BBB in the early stages of the disease. At an early stage of the disease, as they invade the blood vessels, the tumor cells are not completely protected by the BBB, thus making them more vulnerable to targeted drugs delivered via the bloodstream to the brain. If the above findings hold true in humans, treatment with anti-invasive agents might be beneficial in newly diagnosed GBM patients.

h) Self-eradication during meiosis: During anaphase in mitosis, an "inherent death mechanism" can self-eradicate duplicating cancer cells without impairing healthy cells in both normally and rapidly proliferating human cancer cells. The faster cancer cells proliferate, the faster and more efficiently they will be eradicated. The mechanism involves the modification of specific proteins that affect the construction and stability of the spindle, a newly discovered mechanism is able to arrest cancer cells from dividing and multiplying, thus stopping cancer progression in its track. It may be suitable for treating aggressive cancers that are not responsive to traditional chemotherapy. Three proteins can be specifically modified during mitosis to unleash an "inherent death mechanism". Certain compounds (the phenanthridine derivatives) are able to impair the activity of these proteins, distorting the spindle structure and preventing the segregation of chromosomes. Once the proteins are modified, the cells are prevented from dividing, inducing the cell's rapid self-destruction and apoptosis. A variety of additional drugs that also modify these specific proteins could be developed.

i) Inflammation: Many malignancies arise in areas of chronic inflammation and inadequate resolution of inflammation could have a major role in tumor invasion, progression, and metastasis although it may not play a role in oncogenesis. Inflammation is of particular pathophysiological relevance in lung cancer in that chronic bronchitis, triggered by asbestos, silica, smoking, and other external inhaled toxins, results in a persistent inflammatory response. Inflammation in the tumor microenvironment mediated by interleukin IL-1 $\beta$ has a major role in cancer invasiveness, progression, and metastasis. This, in turn, suggests that IL-1 $\beta$ participates in the invasiveness of already existing malignancies. Thus, inhibition of IL- $1 \beta$ might have an adjunctive role in the treatment of cancers that have at least a partial inflammatory basis. Thus, inhibition of IL- $1 \beta$ with the monoclonal antibody Canakinumab is associated with reduced incidences of fatal cancer, lung cancer, and fatal lung cancer. Anti-inflammatory therapy with Canakinumab targeting the IL- $1 \beta$ innate immunity pathway could significantly reduce incident lung cancer and lung cancer mortality.

j) Electrochemotherapy: Electrochemotherapy (ECT) delivers non-permeant drugs (e.g., bleomycin) or low-permeant drugs (e.g., cisplatin, an alkalyting agent) to the cell interior. The technique is based on the local application of short and intense electric pulses that transiently permeabilize the cell membrane, thus allowing the transport of chemotherapeutic molecules through it (a phenomenon called electroporation or electropermeabilization). The pulse characteristics (amplitude, phase, pulse duration and repetition frequency, DC- or ACcurrents, shape and position of the electrodes) are chosen depending on the tissues; however, the pulse amplitude has to be high enough to establish a $400 \mathrm{Volt} / \mathrm{cm}$ electrical field in the tumor area and yield eight $100 \mathrm{~ms}$ pulses at a $5000 \mathrm{~Hz}$ repetition frequency for patient comfort and shorter treatment duration. For deep-seated tumors in the relative vicinity of the heart, pulses are synchronized with the absolute refractory period of each heart beat so as to minimize the probability of their interaction with the heart function. The drugs can then freely diffuse into the cytoplasm and exert their cytotoxic effect. ECT has been widely used for cutaneous and subcutaneous tumors or their metastases, yielding an objective response rate $>80 \%$ and, when compared to standard chemotherapy, a faster and more efficient tumor size reduction. Patients with skin metastasis from melanoma, Kaposi sarcoma, squamous cell carcinoma, basal cell carcinoma, adenocarcinoma, or breast cancer have been successfully treated. ECT employs lower dosages of chemotherapeutic drugs than standard chemotherapy protocols resulting in lower patient's burden and more limited side effects.

k) Nanochemotherapy: Nanochemotherapy (NCT) uses nanodevices (ND) to deliver nanoparticles (NP) containing cytotoxic drugs to tumors. NPs include nutshells $(120 \mathrm{~nm}$ in diameter coated with gold), platelet-coated NPs $(\sim 100 \mathrm{~nm}$ in diameter), gelatin NPs for delivering multiple drugs to the brain, shape-shifting engineered NPs, , bioavailabilityimproved nanoscale particles and molecules, and others. The basic process to use drug delivery here involves at least three steps: (a) encapsulation of the drugs; (b) successful delivery of said drugs to the targeted region of the body; and (c) successful release of that drug there. The drugs are delivered employing engineered NDs. Because of their diverse capabilities, NDS can contain both targeting and therapeutic agents (in both single and multi-drug approaches). They can deliver high drug levels in several situations, including anticancer drugs at the tumor site that can increase chemotherapeutic efficacy. They can also be "smart" nanotherapeutics to "time" the release of any given drug or to deliver multiple drugs sequentially in a timed manner or at several locations in the body. There are numerous clinical advantages: the nNPs circulate throughout the bloodstream without being attacked by the immune system; they preferentially bind to damaged blood vessels and certain pathogens such as MRSA (Methycillin Resistant Staphylococcus Aureus) bacteria, allowing them to deliver and release their drug payloads specifically to these body sites in the body; they are non-toxic as the platelet membranes are nanoparticle cores made of a biodegradable polymer that can be safely metabolized by the body; and they can be packed with many small drug molecules that diffuse out of the polymer core and through the platelet membrane onto their targets.

With the momentous advances of the Human Genome Project (HGP) and its sequel the Human Cancer Genome Project (HCGP), we have now come to the realization that understanding more intimately the biology of cancer, gene by gene and pathway by pathway, will direct us into the right direction for cancer therapeutics. (A second direction would obviously be cancer prevention, or at least prevention of those factors that may trigger cancer. The third and last direction would be to integrate our understanding of aberrant genes and pathways to explain the behavior of cancer.) This would lead to a personalized approach to cancer treatment along the lines advocated 
for a new paradigm in medicine and health care, namely, personalized medicine. This brings us to "oncogenomics" as the newest approach to cancer treatment.

\section{The oncogenomic approach}

With the above in mind, we are now searching for all genomic processes and pathways that could evidence actionable treatment indicators utilizing a multi-drug chemotherapeutic tool. Unfortunately, as a sequel to various chemotherapies, patients develop resistance to drugs and therapies and, left without options, those who relapse enroll in a succession of generally unsuccessful clinical trials because they lack a clear biological rationale to guide them. Additionally, other cancer theories and therapies continue to be proposed.

Research about cancer causes now focuses on the following issues:

i. What are the agents (e.g. viruses) and events (e.g. mutations) that cause or facilitate genetic changes in cells destined to become cancerous?

ii. What is the precise nature of the genetic damage, and the genes that are affected by it? And

iii. What are the consequences of those genetic changes on the biology of the cell, both in (a) generating the defining properties of a cancer cell, and (b) facilitating additional genetic events that lead to further progression of the cancer.

We distinguish between solid and non-solid (e.g., blood) tumors. In the former case, immunotherapy with autologous CAR-T cells engineered to target the appropriate cancer cells is employed. Standard DNA tests are conducted to zoom in on specific loci in the cancer cells' genome. Such DNA tests have been useful in identifying "actionable mutations", that is, those that indicate the cells could be vulnerable to a particular drug. The idea of basing cancer treatments on DNA mutations is not new although it can be difficult to sift out "actionable findings" from DNA sequencing data. In non-solid tumors (e.g., myeloma, a blood cancer): autologous CAR-T cells engineered to target RCMA (a protein on the surface of myeloma cells) did not succeed. Research on multiple myeloma has lagged behind solid tumors in trying to implement a personalized approach and incorporating genomics data in the treatment.

Problems with multiple myeloma are multiple in that pathology reports do not identify clearly the drivers of the cancer and DNA sequencing does not always give a clue as to how to manage the cancer. Possible solutions are then RNA sequencing to have a peek not just at gene mutations, but also at other changes in the cancer cells (example: copy number variations, $\mathrm{CNV}$ ) that might be treatmentrelevant. The most recent application of genomics to cancer treatment looking beyond DNA has been conducted for multiple myeloma by a New York Mt Sinai team of researchers (Parekh, Perumal et al.). Their trial was limited and included 64 of the hospital's patients who had relapsed or not been responding to standard treatments. Cancer cells' DNA and messenger RNA were sequenced and searched for anything that might respond to any of the approved cancer drugs (even if not for multiple myelomas). Only 26 subjects received the recommended personalized treatment (other patients have died or enrolled in other drug trials, and for one patient no drug could be recommended). Out of the 21 evaluable subjects, 16 responded to treatment with the 5-drug cocktail (DCTVI), one of whom experienced complete remission, and 5 had side effects (fatigue or diarrhea). [DCTVI stands for dexomethasone (an anti-inflammatory corticosteroid) + carfizomib (a myeloma treatment) + trametinib (a myeloma treatment) + venetoclax (approved for chronic lymphocytic leukemia) + ibrance (a breast cancer drug)]. The other 5 patients did not stay on the recommended treatment for long enough or did not complete the scans or/and the tests needed for evaluation. ${ }^{1-14}$

\section{Conclusion}

Oncogenomics, that is the personalized cancer therapy, has come of age. While it proceeds from a scanning of the genome of each individual patient for actionable treatment indicators, it uses this information to apply well-known treatment approaches that had heretofore been applied generally with little or no consideration of the treated individual. We are at the initial step where the genome has been scanned for copy number variants. Other steps forward would consist in investigating other genomic information including the transcriptome and reverse transcriptome, and mechanisms of genetic changes (configuration, deletion, disruption, insertion, point mutation, replication, and translocation). This application may be time and resource prohibitive but this process could and should be accelerated.

\section{Acknowledgements}

None.

\section{Conflict of Interest}

Author declares that there is no conflict of interest.

\section{References}

1. Fymat AL. The Long Quest for Cancer Cures. J Cancer Prev Curr Res. 2016;6(2):1-3.

2. Fymat AL. Recent Research Developments in Anti-Cancer Therapy. $J$ Cancer Prev Curr Res. 2016;5(2):1-2.

3. Fymat AL. Antiangiogenic Targeting of Early Developing Glioblastoma Behind a Weakened Blood Brain Barrier. Journal of Anti-Tumor Medicine \& Prevention. 2017;2(3):1-6.

4. Fymat AL. Disrupting Cell Mitoses to Provoke Cancer Self-Destruction. Cancer Therapy \& Oncology International Journal. 2017;5(1):1-4.

5. Fymat AL. On the Inflammation Theory of Cancer. Cancer Therapy \& Oncology International Journal. 2017;8(3):1-7.

6. Fymat AL. On Cancer's Theories, True Nature, and Possible SelfEradication. Cancer Therapy \& Oncology International Journal. 2017;7(2):1-3.

7. Fymat AL. Genetics, Epigenetics and Cancer. Cancer Therapy \& Oncology International Journal. 2017;4(2):1-11.

8. Fymat AL. Anti-Tumor Therapies: Cases of Breast and Prostate Cancers. Journal of Tumor Medicine \& Prevention. 2017;1(2):1-12.

9. Fymat AL. Glioblastoma Treatments: Where Do We Stand?. MedPlus Journal of Cancer \& Oncology Research. 2017;1(1):1-12.

10. Fymat AL. Surgical and Non-Surgical Management and Treatment of Glioblastoma: I. Primary Tumors. Open Access Journal of Surgery. 2017;7(2):1-8.

11. Fymat AL. Surgical and Non-Surgical Management and Treatment of Glioblastoma: II. Recurring Tumors. Open Access Journal of Surgery. 2017;7(1):1-7.

12. Fymat AL. Innate Immunotherapy of Recurring Glioblastoma: Preliminary Trials with Neutrophils. Journal of Current Opinions in Neurological Science. 2018;2(3):480-482.

13. JCO. Precis Oncol. 2018;doi:10.1200/PO.18.00019.

14. Parekh S, Perumal D. Omics-ing Cancer. The Scientist 16-7, 2018. 